Tropical Journal of Pharmaceutical Research June 2017; 16 (6): 1239-1244

ISSN: $1596-5996$ (print); 1596-9827 (electronic)

(C) Pharmacotherapy Group, Faculty of Pharmacy, University of Benin, Benin City, 300001 Nigeria.

All rights reserved.

Available online at http://www.tjpr.org

Original Research Article

http://dx.doi.org/10.4314/tjpr.v16i6.5

\title{
Herbal carrier-based floating microparticles of diltiazem hydrochloride for improved cardiac activity
}

\author{
Yun Zhou', Min-Li Zhang', Bai-Jing Yuan', Jin-qi Yuan', Xin-Fang Zhao', Li \\ Zhao $^{2}$ and Hong-Qiang Ren ${ }^{3 *}$ \\ ${ }^{1}$ Department of Traditional Chinese Medicine, ${ }^{2}$ Department of Emergency Internal Medicine, ${ }^{3}$ Department of Cardiology, The \\ First Affiliated Hospital, Medical College of Shihezi University, Xinjiang 832008, PR China
}

${ }^{*}$ For correspondence: Email: renhongqiang256@hotmail.com; Tel/Fax: 0086-0993-2860314

Sent for review: 3 September 2016

Revised accepted: 6 May 2017

\begin{abstract}
Purpose: To formulate and characterize a gastroretentive floating drug delivery system for diltiazem hydrochloride using psyllium husk and sodium alginate as natural herbal carriers to improve the therapeutic effect of the drug in cardiac patients.

Methods: Floating microparticles containing diltiazem hydrochloride were prepared by the orifice ionic gelation technique. Various physicochemical properties of the floating microspheres were characterized, including drug content, particle size, surface morphology, in vitro drug release, and in vivo antihypertensive effect.

Results: The diltiazem hydrochloride microparticles exhibited a high drug content ranging from $63.23 \pm$ 1.14 to $85.56 \% \pm 1.14 \%$. The particle size was $891.40 \pm 2.14,928.40 \pm 1.79,900.65 \pm 2.22$, and $1345.40 \pm 1.36 \mu \mathrm{m}(p<0.05$ compared to blank microspheres for formulations FDD1, FDD2, FDD3, and FFD4), respectively. Scanning electron microscopy showed that all the formulations had a smooth spherical surface with little pores and few cracks. The maximum floatability value was $83.11 \% \pm 3.18 \%$ for FDD1. All of the formulations showed good in vitro drug release profiles, with a maximum release of $87.4 \%$ of the drug at the end of 12 hours. The in vivo antihypertensive effects of the microparticles in human subjects were significant ( $p<0.05$ compared to normal controls), with a reduction in diastolic blood pressure from 120 to $78 \mathrm{mmHg}$ at the end of 4 hours compared to diltiazem sustained-release tablets.

Conclusion: Psyllium husk and sodium alginate-based microspheres can be suitably prepared for the controlled delivery of diltiazem hydrochloride to cardiac patients. However, further study is required to develop the delivery system.
\end{abstract}

Keywords: Diltiazem, Cardiac disease, Psyllium husk, Sodium alginate, Microsphere, Microparticle, Controlled drug release, Gastroretentive, Floating drug delivery

Tropical Journal of Pharmaceutical Research is indexed by Science Citation Index (SciSearch), Scopus, International Pharmaceutical Abstract, Chemical Abstracts, Embase, Index Copernicus, EBSCO, African Index Medicus, JournalSeek, Journal Citation Reports/Science Edition, Directory of Open Access Journals (DOAJ), African Journal Online, Bioline International, Open-J-Gate and Pharmacy Abstracts

\section{INTRODUCTION}

Herbal-based drug delivery systems have long been used in folk medicine, and herbal medicines of natural origin show good therapeutic activity with minimal side effects. The World Health Organization estimates that $80 \%$ of the world's population currently uses herbal medicines for primary health care. Thus, researchers have begun to focus on herbal drugs and the use of materials of herbal origin. Herbal medicines have many advantages over traditional medicines, including a lower risk of side effects, lower cost, and widespread availability $[1,2]$. 
Gastroretention is the most feasible approach for achieving prolonged, predictable, and controlled drug delivery in the gastrointestinal tract. These systems have a lower density than gastric fluid and remain buoyant in the stomach without affecting gastric emptying for prolonged periods [3-6].

Psyllium husk is a source of mucilaginous fiber obtained from the dried seed coats of Plantago ovata. It forms a gel upon contact with water, and its swelling behavior makes it suitable for use as a carrier for diltiazem hydrochloride $(\mathrm{HCl})$ microparticles $[7,8]$.

Diltiazem $\mathrm{HCl}$ is a calcium channel blocker that inhibits the influx of calcium $\left(\mathrm{Ca}^{2+}\right)$ ions during membrane depolarization in cardiac and vascular smooth muscle. It is effective in the treatment of hypertension and angina pectoris due to its cardiac effects. It has $40 \%$ oral bioavailability [9], and so requires frequent dosing to avoid these drawbacks. The administration of conventional diltiazem $\mathrm{HCl}$ tablets causes fluctuations in the plasma drug level resulting in side effects due to reduced drug concentrations at receptor sites. Furthermore, the maintenance of a constant plasma concentration of cardiac vascular drugs is important to ensure the designed therapeutic response; as the half-life of diltiazem $\mathrm{HCl}$ is 3 $4 \mathrm{~h}$, multiple doses of the drug are needed to maintain a constant plasma concentration for a good therapeutic response and to improve patient compliance [10-13].

The present study was performed to develop a gastroretentive floating drug delivery system for diltiazem $\mathrm{HCl}$ using psyllium husk and sodium alginate as natural herbal carriers to achieve improved therapeutic effects in cardiac patients.

\section{EXPERIMENTAL}

\section{Materials}

Diltiazem $\mathrm{HCl}$ was obtained from Sigma-Aldrich (St. Louis, MO). Psyllium husk was obtained from the Department of Traditional Chinese Medicine, Medical College of Shihezi University, Xinjiang, China. All other reagents were of analytical grade or higher and obtained commercially.

\section{Preparation of diltiazem HCl microparticles}

Floating microparticles containing diltiazem $\mathrm{HCl}$ were prepared by the orifice ionic gelation technique using the composition shown in Table 1.
Table 1: Composition of microparticles of diltiazem $\mathrm{HCl}(\mathrm{DHCl})$

\begin{tabular}{lccc}
\hline Formulation & $\begin{array}{c}\text { DHCl } \\
(\mathbf{m g})\end{array}$ & $\begin{array}{c}\text { Sodium } \\
\text { alginate } \\
(\% \mathbf{\%} / \mathbf{v})\end{array}$ & $\begin{array}{c}\text { Psyllium } \\
\text { husk } \\
(\% \mathbf{\%} / \mathbf{v})\end{array}$ \\
\hline $\mathrm{FDD}_{1}$ & 100 & 200 & 150 \\
$\mathrm{FDD}_{2}$ & 100 & 100 & 150 \\
$\mathrm{FDD}_{3}$ & 100 & 150 & 100 \\
$\mathrm{FDD}_{4}$ & 100 & 250 & 50 \\
\hline
\end{tabular}

Sodium alginate and psyllium husk were dissolved in purified water to form a firm gel, and the gas-forming agent sodium carbonate was dispersed in the purified water to form a homogeneous polymer mixture. The drug was added to the polymeric solution and mixed thoroughly with a magnetic stirrer to form a homogeneous mixture. Gelation medium containing $2 \%$ calcium chloride in glacial acetic acid was prepared. The homogenous polymer solution was extruded into the gelation medium using a 21-G needle. The distance between the tip of the needle and surface of the gelation medium was maintained at $10 \mathrm{~cm}$. The mechanical strength of the microparticles was improved by gentle stirring for 30 minutes at room temperature. The prepared microparticles were collected, washed twice with distilled water, dried at room temperature for 24 hours, and stored in desiccators [14].

\section{Assessment of microparticle size and shape}

The size of the microparticles was determined using an optical microscope fitted with an ocular and stage micrometer. Scanning electron microscopy (SEM) was performed to determine the surface morphology of the formed microparticles. The preparations were mounted directly onto the sample stub and coated with gold film ( 200 nm) under reduced pressure $(0.130 \mathrm{~Pa})$ (JSM-1600; JEOL Ltd., Tokyo, Japan).

\section{Determination of drug content}

The drug content was determined by dissolving a $50 \mathrm{mg}$ equivalent of diltiazem $\mathrm{HCl}$ microspheres in $100 \mathrm{~mL}$ of $0.1 \mathrm{~N} \mathrm{HCl}$.

This solution was then stirred on a magnetic stirrer for 24 hours and filtered. Next, the samples were withdrawn, filtered, diluted appropriately, and measured spectrophotometrically at $243 \mathrm{~nm}$ to determine the drug content. 


\section{Evaluation of bulk density}

The bulk density of the prepared gastroretentive floating microspheres was determined using a standard bulk density apparatus.

\section{Buoyancy test}

In vitro floating behavior studies were performed by placing 50 microparticles in $50 \mathrm{~mL}$ glass flasks. To exclude floating due to non-wet surfaces, an additional $50 \mathrm{~mL}$ of $0.1 \mathrm{~N} \mathrm{HCl}$ containing $0.02 \%$ w/v Tween 20 was added followed by horizontal shaking $\left(37^{\circ} \mathrm{C}, 75 \mathrm{rpm}\right)$. The flasks were allowed to stand for 5 minutes without agitation, and the particles were counted. This procedure was repeated after 2, 4, 6, and 8 hours. The percentage of floating microspheres was calculated using the following equation.

\section{In vitro drug release studies}

The in vitro release profile of the microspheres was evaluated using an eight-station USP dissolution test apparatus with $900 \mathrm{~mL}$ of acid buffer ( $\mathrm{pH}$ 1.2) as the dissolution medium. It was maintained at $37 \pm 0.5{ }^{\circ} \mathrm{C}$ at $50 \mathrm{rpm}$. Aliquots of an accurately weighed $100 \mathrm{mg}$ equivalent of drug microspheres were added and dissolution was performed for $2.5 \mathrm{~h}$. Samples (5 $\mathrm{mL}$ each) were withdrawn every 30 minutes and replaced with an equal volume of fresh medium to maintain sink conditions. The samples were filtered, diluted appropriately, and analyzed spectrophotometrically for drug release at $243 \mathrm{~nm}$. The dissolution medium was then replaced by $900 \mathrm{~mL}$ of phosphate buffer ( $\mathrm{pH}$ 6.8) maintained at $37^{\circ} \mathrm{C} \pm 0.5^{\circ} \mathrm{C}$ at $50 \mathrm{rpm}$. This study was continued for $12 \mathrm{~h}$.

\section{In vivo antihypertensive effect}

This study was conducted in accordance with the International Ethical Guidelines for Biomedical Research Involving Human Subjects [15]. The study was approved by the ethics committee of Medical College of Shihezi University (approval no. TEK-1345523). The antihypertensive effect was studied in 60 hypertensive men aged $35-$ 45 years with a diastolic pressure of $100-120$ $\mathrm{mmHg}$. Each subject provided written consent prior to participation. The exclusion criteria included patients with obesity, patients on multidrug therapy, liver or renal failure patients, and smokers. The in vivo antihypertensive effect of the selected formulation $(50 \mathrm{mg}$ of diltiazem containing an equivalent amount of microspheres in capsules of size 000) was examined in 30 hypertensive men in comparison to a group of 30 hypertensive men administered oral diltiazem tablets $(10 \mathrm{mg})$. The patients were administered the sample preparation (with about $250 \mathrm{~mL}$ of water) or tablets in the morning after an overnight fast. The diastolic blood pressure was measured in $\mathrm{mmHg}$ at different time intervals (Figure 2).

\section{Statistical analysis}

The results are expressed as mean \pm standard deviation (SD) and were analyzed by Student's ttest. The level of statistical significance was set at $p<0.05$.

\section{RESULTS}

The drug content of the microsphere formulations are show in Table 2. The bulk densities of all formulations indicate good flow properties and floatability. The bulk density was between 0.177 \pm 0.0322 and $0.232 \pm 0.0232 \mathrm{~g} / \mathrm{mL}$.

SEM showed that all of the formulations had a smooth spherical surface with little porosity and few cracks (Figure $1 \mathrm{~A}$ to Figure 1D).

A buoyancy study showed an optimum floating time of more than 8 hours for the formulations. The percentage buoyancy was $83.11 \pm 3.18$, $76.34 \pm 1.15,79.99 \pm 1.89$ and $75.22 \pm 1.44 \%$ for $F D_{1}, F_{2 D}, F D D_{3}$, and $F F D_{4}$, respectively.

At the end of $12 \mathrm{~h}$, the percentage of the drug that had been released was $87.4,80.3,83.1$ and $75.2 \%$ for $\mathrm{FDDD}_{1}, \mathrm{FDD}_{2}, \mathrm{FDD}_{3}$, and $\mathrm{FDD}_{4}$, respectively (Figure 2).

The in vivo antihypertensive results showed that the diastolic blood pressure decreased from 120 to $78 \mathrm{mmHg}$ at the end of 4 hours and maintained this level for 10 hours. On the other hand, diltiazem tablets reduced the patients'

Table 2: Physical characteristics of the diltiazem $\mathrm{HCl}$ microspheres

\begin{tabular}{lcccc}
\hline Code & $\begin{array}{c}\text { Drug content } \\
(\%)\end{array}$ & $\begin{array}{c}\text { Particle size } \\
(\boldsymbol{\mu m})\end{array}$ & $\begin{array}{c}\text { Bulk density }^{\star} \\
\mathbf{g} / \mathbf{m L}(\boldsymbol{\rho b})\end{array}$ & Buoyancy (\%) \\
\hline $\mathrm{FDD}_{1}$ & $85.56 \pm 1.14$ & $891.40 \pm 2.14^{\mathrm{a}}$ & $0.232 \pm 0.0232^{\mathrm{a}}$ & $83.11 \pm 3.18^{\mathrm{a}}$ \\
$\mathrm{FDD}_{2}$ & $80.12 \pm 1.24$ & $928.40 \pm 1.79^{\mathrm{a}}$ & $0.210 \pm 0.0322^{\mathrm{a}}$ & $76.34 \pm 1.15^{\mathrm{a}}$ \\
$\mathrm{FDD}_{3}$ & $81.77 \pm 3.11$ & $900.65 \pm 2.22^{\mathrm{a}}$ & $0.179 \pm 0.0172^{\mathrm{a}}$ & $79.99 \pm 1.89^{\mathrm{a}}$ \\
$\mathrm{FDD}_{4}$ & $63.23 \pm 1.14$ & $1345.40 \pm 1.36^{\mathrm{a}}$ & $0.177 \pm 0.0322^{\mathrm{a}}$ & $75.22 \pm 1.44^{\mathrm{a}}$ \\
\hline${ }^{\star}$ All values are expressed as means $\pm \mathrm{SD}, n=3 ;^{\mathrm{a}} p<0.05$ compared to blank microspheres
\end{tabular}

${ }^{*}$ All values are expressed as means $\pm \mathrm{SD}, n=3 ;{ }^{a} p<0.05$ compared to blank microspheres 



1B

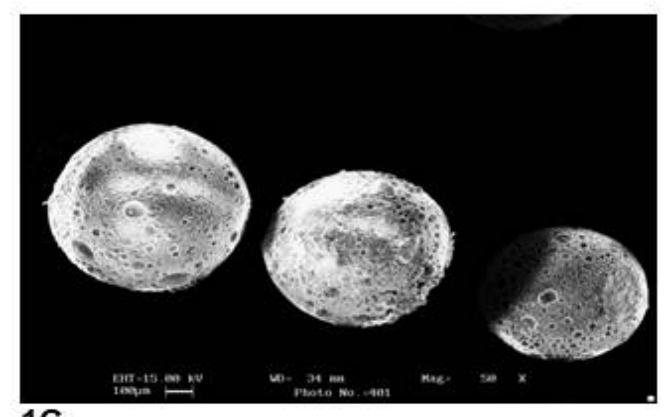

$1 \mathrm{C}$

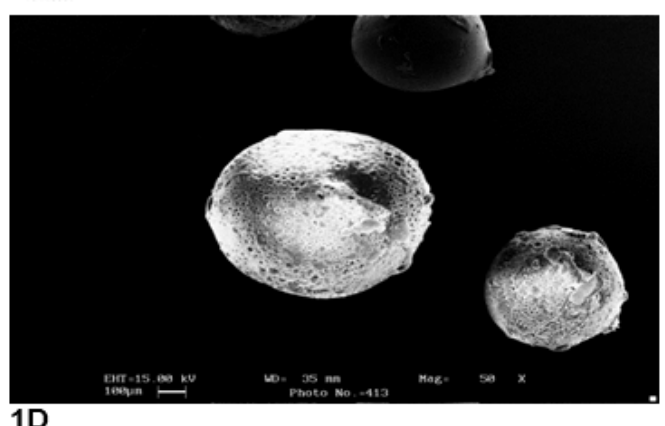

Figure 1: Scanning electron micrographs of floating microspheres: (1A) FDD 1 (50x); (1B) FDD 2 (25x); (1C) $\mathrm{FDD}_{3}(50 \mathrm{x})$; and (1D) $\mathrm{FDD}_{4}(50 \mathrm{x})$

diastolic pressure from 110 to $97 \mathrm{~mm} \mathrm{Hg}$ at the end of 4 hours (Figure 3 ).

\section{DISCUSSION}

Prolonged release of diltiazem from floating microparticles not only increased the therapeutic efficacy and patient compliance but also produced a more reliable plasma drug profile as compared to diltiazem tablets.



Figure 2: In vitro drug release profiles for floating microspheres of diltiazem: $F_{1}(-\bullet-), F_{2}(-\square-), F_{3}(-\mathbf{\Delta})$, and $\mathrm{F}_{4}(-\circ-)$

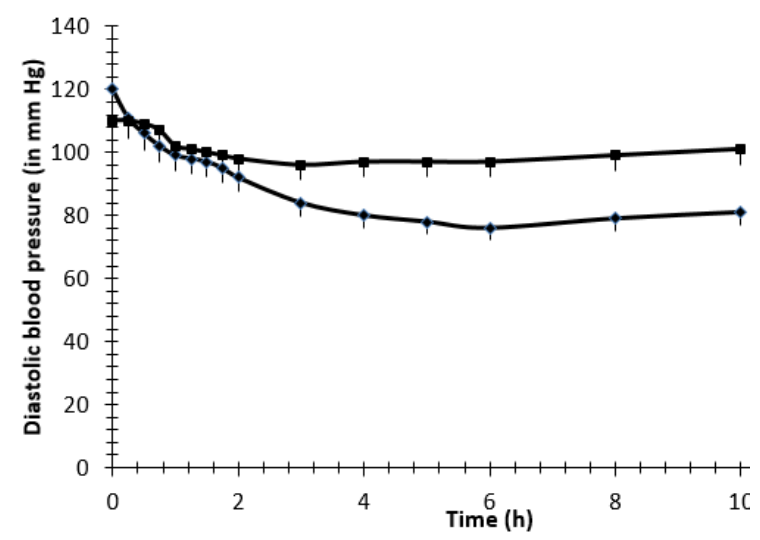

Figure 3: In vivo antihypertensive effect of floating microsphere formulation $\mathrm{FDD}_{2}(-\bullet)$ vs. conventional tablets (-a-)

The particles ranged in size from $891.40 \pm 2.14$ to $1345.40 \pm 1.36 \mu \mathrm{m}$. The mean particle size varied significantly according to the different proportions of polymers used, which may have been due to differences in the viscosity of the polymer solution [16-20]. The particles in formulation $\mathrm{FDD}_{1}$ (containing a greater quantity of psyllium husk) were larger than those in the other formulations. This is not surprising since psyllium husk contributed significantly to the viscosity of the polymer solution. Previous studies also suggested that psyllium husk-based extended release drug preparations were effective in delivering the loaded drugs. An increase in psyllium husk concentration was shown to increase both the viscosity of the dispersed phase and the entrapment efficiency of microparticles [21-23].

All of the formulations showed significant drug loading, which may have been due to strong intermolecular bonding between the polymer and drug. Thus, the drug was uniformly distributed and an optimum drug concentration was obtained at the site of action. 
Determinations of bulk density are important with respect to floating drug delivery to ensure good flow properties and floatability. Previous studies also suggested the floating behavior of diltiazem microspheres (maximum floatability: $85.31 \%$ ). As the size of the microparticles increased, the bulk density decreased due to an increase in space between the molecules.

In vitro buoyancy testing showed a maximum percent buoyancy of $83.11 \pm 3.18 \%$ at the end of 8 hours. The good floating behavior of the particles was attributed to the low density and hollow nature of the microparticles. Previous studies also suggested that hollow cavities in microparticles occurred due to the production of air bubbles during their formulation.

The formulations yielded a gradual and more sustained release of the drug over the study period. These results show that diltiazem-loaded microparticles could be used once daily for the treatment of arrhythmia, angina, and other cardiac disorders. In the first few hours, the microspheres provided an initial burst of release due to rapid dissolution of the drug from the surface of the microparticles $[24,25]$. This can be considered as the loading dose, with the rest of the drug release considered as the maintenance dose. The release kinetics indicates that the drug release from the formulations followed a matrix diffusion process. Based on the obtained particle size, morphology, drug content, in vitro release, and release kinetics, formulation $\mathrm{FDD}_{1}$ was selected for testing of its in vivo antihypertensive effects. The results confirm that FDD $_{1}$ efficiently reduced the patients' diastolic blood pressure from 120 to $78 \mathrm{mmHg}$ at the end of $4 \mathrm{~h}$, in comparison with diltiazem tablets. In vitro drug release, buoyancy and in vivo antihypertensive data indicate that formulation $\mathrm{FDD}_{1}$ would reside in the gastric region for a long period and thus increase drug absorption.

\section{CONCLUSION}

Psyllium husk and sodium alginate have been used as natural herbal carriers for formulations of diltiazem floating microparticles to achieve drug release in the treatment of hypertension. The floating microparticles display good floating, suitable in vitro drug release profile and good in vivo antihypertensive effects. Thus, the formulation has potential for use as a drug delivery system, but additional studies are required to confirm these findings.

\section{DECLARATIONS}

\section{Acknowledgement}

The authors thank The First Affiliated Hospital, Medical College of Shihezi University for providing the necessary facilities to conduct this research.

\section{Conflict of Interest}

No conflict of interest associated with this work.

\section{Contribution of Authors}

The authors declare that this work was done by the authors named in this article and all liabilities pertaining to claims relating to the content of this article will be borne by them.

\section{Open Access}

This is an Open Access article that uses a funding model which does not charge readers or their institutions for access and distributed under the terms of the Creative Commons Attribution License (http://creativecommons.org/licenses/by/ 4.0) and the Budapest Open Access Initiative (http://www.budapestopenaccessinitiative.org/rea d), which permit unrestricted use, distribution, and reproduction in any medium, provided the original work is properly credited.

\section{REFERENCES}

1. Acharya SN, Parihar VG, Acharya RS. Hytosomes: novel approach for delivering herbal extract with improved bioavailability. Int J Pharm Sci 2011; 2: 144-160.

2. Alzaher $W$, Shaw J, Al-Kassas R. Gastroretentive formulations for improving oral bioavailability of drugsfocus on microspheres and their production, Curr Drug Deliv 2016; 13(5): 646-661.

3. Bhadouriya $P$, Kumar $M$, Pathak $K$. Floating microspheres: to prolong the gastric retention time in stomach. Curr Drug Deliv 2012; 9(3): 315-324.

4. Soppimath KS, Kulkarni AR, Rudzinski WE, Aminabhavi TM. Microspheres as floating drug-delivery systems to increase gastric retention of drugs. Drug Metab Rev 2001; 33(2): 149-1460.

5. Jiang $H$, Tian $R$, Hu W, Jia $Y$, Yuan $P$, Wang J, Zhang $L$. Formulation and evaluation of gastroretentive floating drug delivery system of dipyridamole. Drug Devind Pharm 2015; 41(4): 674-680.

6. Dhar, MK, Kaul S, Sareen S, Koul AK. Plantago Ovata: genetic diversity, cultivation, utilization and chemistry. Plant Genetic Resources 2005; 3: 252-263.

7. Fischer $M H, Y u N X$, Gray GR, Ralph J, Anderson L, Marlett JA. The gel-forming polysaccharide of psyllium 
husk (PlantagoovataForsk). Carb Res 2004; 339: 2009 2017.

8. Sultana $Y$, Mall $S$, Maurya $D P$, Kumar $D$, Das $M$. Preparation and in vitro characterization of diltiazem hydrochloride loaded alginate microspheres. Pharm Dev Technol 2009; 14(3): 321-331.

9. Das MK, Maurya DP. Evaluation of diltiazem hydrochloride-loaded mucoadhesive microspheres prepared by emulsification-internal gelation technique. Acta Pol Pharm 2008; 65(2): 249-259.

10. Semalty A, Bhojwani M, Srivastav AK, Gupta GD. Design and evaluation of mucoadhesive buccal films of diltiazem hydrochloride. Indian J Pharm Sci 2005; 67(5): 548-552.

11. Srivastava A, Gowda DV, Hani U, Shinde CG. Design of a porous chitosan based matrix tablet as carrier for controlled delivery of diltiazem hydrochloride. J Biomater Tissue Eng 2014; 4: 804-810.

12. Sun $C$, Liu $H$, Zhao $X, H e H, P a n W$. In vitro and in vivo evaluation of a novel diltiazem hydrochloride polydispersity sustained-release system. Drug Dev Ind Pharm 2013; 39(1): 62-66.

13. Cnoi BY, Park J, Hwang SJ, Park JB. Preparation of alginate beads for floating drug delivery system: effects of $\mathrm{CO}_{2}$ gasforming agents. Int $\mathrm{J}$ Pharm 2002; 239: 81 91.

14. Foster CG. International ethical guidelines for biomedical research involving human subjects. J Med Ethics 1994; 20(2): 123-124

15. Kiliçarslan M, Baykara T. The effect of the drug/polymer ratio on the properties of the verapamil $\mathrm{HCl}$ loaded microspheres. Int J Pharm 2003; 252(1-2): 99-109.

16. Pongpaibal Y, Price JC, Whitworth CW. Preparation and evaluation of controlled release indomethacine microspheres. Drug Dev Ind Pharm 1984; 10: 1597 1616.
17. Barkai A, Pathak YV, Benita S. Polyacrylate (Eudragit Retard) microspheres for oral controlled release of nifedipine. I. Formulation design and process optimization. Drug Dev Ind Pharm 1990; 16: 2057-2075.

18. Bijanzadeh M, Mahmoudian M, Zolfaghari ME, Gouya MM, Khazinia TK. The influence of particle size and dissolution rate on bioavailabilty of two indomethacine capsules. DARU 1995; 55: 51-61.

19. Bogataj M, Mrhar A, Krist A, Kozjek F. Eudragit $E$ microspheres containing bacampicillin: preparation by solvent removal methods. J Microencapsul 1991; 8: 401-406.

20. Mukherjee B, Santra K, Pattnaik G, Ghosh S. Preparation, characterization and in-vitro evaluation of sustained release protein-loaded nanoparticles based on biodegradable polymers. Int J Nanomedicine 2008; 3(4): 487-496.

21. Singh B, Chauhan N. Dietary fiber psyllium based hydrogels for use in insulin delivery. Int J Diab Mell 2010; 2 (1): 32-37.

22. Cheng HF, Feng $Y$, Duan QJ, Jiang DM, Tao $K Y$. Tropical floating microparticulate oral diltiazem hydrochloride delivery system for improved delivery to heart. J Pharm Res 2015; 14(6): 935-940.

23. Sawyer AA, Song SJ, Susanto E, Chuan P, Lam CXF, Woodruff MA, Hutmacher, DW, Cool SM. The stimulation of healing within a rat calvarial defect by MPCL-TCP/collagen scaffolds loaded with rhBMP-2. Biomaterials 2009; 30: 2479-2488.

24. Woodruff MA, Rath $S N$, Susanto E, Haupt $L M$, Hutmacher DW, Nurcombe V, Cool SM. Sustained release and osteogenic potential of heparansulfatedoped fibrin glue scaffolds within a rat cranial model. $J$ Mol Histol 2007; 38: 425-433. 